\title{
Editorial
}

\section{Advances in Multiphase Flow and Transport in the Subsurface Environment}

\author{
Xiaoqing Shi $\mathbb{D},{ }^{1}$ Stefan Finsterle, ${ }^{2}$ Keni Zhang, ${ }^{3}$ and Dan $\mathrm{Lu}^{4}$ \\ ${ }^{1}$ School of Earth Sciences and Engineering, Nanjing University, Nanjing, China \\ ${ }^{2}$ Finsterle GeoConsulting, Kensington, CA, USA \\ ${ }^{3}$ Lawrence Berkeley National Laboratory, Berkeley, CA, USA \\ ${ }^{4}$ Oak Ridge National Laboratory, Oak Ridge, TN, USA \\ Correspondence should be addressed to Xiaoqing Shi; shixq@nju.edu.cn
}

Received 4 February 2018; Accepted 5 February 2018; Published 4 March 2018

Copyright (C) 2018 Xiaoqing Shi et al. This is an open access article distributed under the Creative Commons Attribution License, which permits unrestricted use, distribution, and reproduction in any medium, provided the original work is properly cited.

Multiphase flow and transport processes in the subsurface environment are extremely important in a number of industrial and environmental applications at various spatial and temporal scales. Thus, it is necessary to identify, understand, and predict these processes to improve the production of conventional and unconventional oil and gas, to increase the safety of geological sequestration of carbon dioxide and nuclear waste disposal, and to make remediation of contaminated aquifers more effective.

The past decades have seen remarkable growth in research related to multiphase flow and transport in porous and fracture media [1-4]. Numerical models have been widely used to understand, predict, and optimize complex physical fluid flow processes combined with chemical, thermal, mechanical, and biological interactions occurring between fluids and the reservoir rocks [5-7]. Nevertheless, theoretical and practical challenges remain considerable. First, advanced mathematical models are needed to robustly and efficiently analyze the vast variety and complexity of multiphase processes. Second, application of these models to actual field site remains challenging, specifically in areas that involved strongly coupled processes, such as compressed gas energy storage, energy recovery from geothermal reservoirs as well as from coalbed methane, and gas hydrate deposits.

This special issue discusses some of the latest research outcomes in two parts: (1) theoretical fundamentals of multiphase flow and transport related numerical methods and laboratory experiments and (2) field applications to complex subsurface environments. We received 24 submissions, 13 of which passed the initial assessment and review process. A brief summary of this issue is given in the following.

For the theoretical fundamentals, we have the following:

(1) The paper by Y. Hou et al. investigated the anomalous dispersion of nonreactive solute particles in immobile zones and estimated the waiting time distribution. The study demonstrates that the distribution of waiting time in different dead-end pores having the similar power-law decline at early time and transiting to an exponential decline in the end. The anomalous dispersion is highly dependent on the sizes of immobile zones.

(2) The paper by Y. Ning et al. discussed the effects on the uranium leaching efficiency of the lignite from the solid/liquid ratio, $\mathrm{CO}_{3}{ }^{2-} / \mathrm{HCO}_{3}{ }^{-}$ratio, the leaching reagent concentration, and oxidants. They found the optimal solid/liquid ratio and $\mathrm{CO}_{3}{ }^{2-} / \mathrm{HCO}_{3}{ }^{-}$ratio that lead to the maximum uranium leaching rate.

(3) The paper by N. Liu et al. presented numerical simulation by an integrated wellbore-reservoir simulator (T2Well) to investigate the leakage problem along open fractures. Darcy's law in open fractures is validated by comparing the momentum model and corresponding EPM model of various apertures.

(4) The paper by J. Huo et al. presented a decoupling approach by linear algebraic manipulations of the stoichiometric coefficients of the chemical reactions 
to reduce the number of equation variables and simplifying the reactive source in the reactive transport model of pyrite oxidative dissolution.

(5) The paper by J. Han et al. conducted numerical simulations to reproduce the bare silt soil drying process to quantitatively understand the evolutions of variables involved in the evaporation process during different stages of evaporation.

(6) The paper by Y. Dai et al. developed an incompressible two-phase (crude oil and water) flow model incorporating interfacial forces and nonzero contact angles to accurately describe the flow characteristic of fracture scale displacements of immiscible fluids.

(7) The paper by Y. Li et al. investigated the influences of the well screen length on compressed air energy storage in aquifers (CAESA) system performance using an integrated wellbore-reservoir simulator T2WELL.

(8) The paper by J. Piao et al. compared coupled wellborereservoir and conventional equivalent porous media models to investigate dynamic behavior of $\mathrm{CO}_{2}$ in a wellbore and storage formation during geologic $\mathrm{CO}_{2}$ sequestration. The study demonstrates the importance of accounting for the complex thermodynamic behavior of $\mathrm{CO}_{2}$ as it travels along the injection well and interacts with the surrounding formation. Coupled wellbore-reservoir effects may strongly affect the conditions near the injection interval and thus the injectivity, salt-precipitation potential, and other factors determining the performance and sustainability of $\mathrm{CO}_{2}$ injection.

For the field applications, we have the following:

(9) The paper by Y. Yu et al. performed reactive transport simulations to analyze the geochemical interactions between $\mathrm{CO} 2$, formation fluid, and host rock of the major formations in the Ordos Basin and assess the CO2 trapping capabilities for the Shenhua CO2 capture and sequestration (CCS) project in China.

(10) The paper by C. Dai et al. performed numerical simulations of production from a shale gas reservoir in the Sichuan Basin in China to analyze the production characteristics. They quantified the relative contributions of free and adsorbed gas to the total production rate. Moreover, they discussed the roles that the matrix, natural fractures, and the stimulated reservoir volume play in the recovery of natural gas, which determines the accuracy with which each of these components needs to be characterized or controlled.

(11) The paper by Y. Yuan et al. conducted multiphase fluid-flow model to investigate the gas production performance from the layered hydrate reservoirs by depressurization at the offshore production test site of the Eastern Nankai Trough. They found that the dissociation zone is strongly affected by the vertical reservoir's heterogeneous structure and shows a unique dissociation front. The beneficial production interval scheme should consider the reservoir conditions with high permeability and high hydrate saturation.

(12) The paper by Q. Ge et al. conducted numerical simulation $\mathrm{Cl}^{-}$in pore waters through the aquitard-aquifer system to understand the transport mechanism of natural tracer migration in North Jiangsu coastal plain, China.

(13) The paper by L. Dong et al. established an evaluation index system to quantitatively assess the risk degree for underground goafs considering uncertain factors in mines. This developed system is further applied in 37 underground goafs in Dabaoshan mine, which provides an efficient guidance to both reduce the accident risk and improve the mining environment.

\section{Acknowledgments}

We are glad to share these papers with relevant communities of interest. We sincerely hope that the information, the methods, and the achievements presented in the above papers will improve the understanding of the complex multiphase flow and transport processes and solve some practical engineering problems. With the growing demands on environmentally sustainable energy production, we believe it is essential to continue improving our fundamental understanding of multiphase flow and transport processes and to advance our predictive simulation capabilities for solving challenging real-world problems. We would like to express our sincere gratitude to colleagues who performed candid and valuable reviews of the original manuscripts.

Xiaoqing Shi Stefan Finsterle Keni Zhang Dan Lu

\section{References}

[1] J. C. Parker, "Multiphase flow and transport in porous media," Reviews of Geophysics, vol. 27, no. 3, pp. 311-328, 1989.

[2] C. T. Miller, G. Christakos, P. T. Imhoff, J. F. McBride, J. A. Pedit, and J. A. Trangenstein, "Multiphase flow and transport modeling in heterogeneous porous media: Challenges and approaches," Advances in Water Resources, vol. 21, no. 2, pp. 77120,1998

[3] C. T. Crowe, Multiphase Flow Handbook, C. T. Crowe, Ed., vol. 59, CRC press, 2005.

[4] Y. S. Wu, Multiphase Fluid Flow in Porous and Fractured Reservoirs, Gulf Professional Publishing, 2015.

[5] R. Helmig, Multiphase Flow and Transport Processes in the Subsurface: A Contribution to the Modeling of Hydrosystems, Springer-Verlag, 1997.

[6] Z. Chen, G. Huan, and Y. Ma, Computational Methods for Multiphase Flows in Porous Media, Society for Industrial and Applied Mathematics, 2006.

[7] A. Prosperetti and G. Tryggvason, Computational Methods for Multiphase Flow, A. Prosperetti and G. Tryggvason, Eds., Cambridge University Press, 2009. 

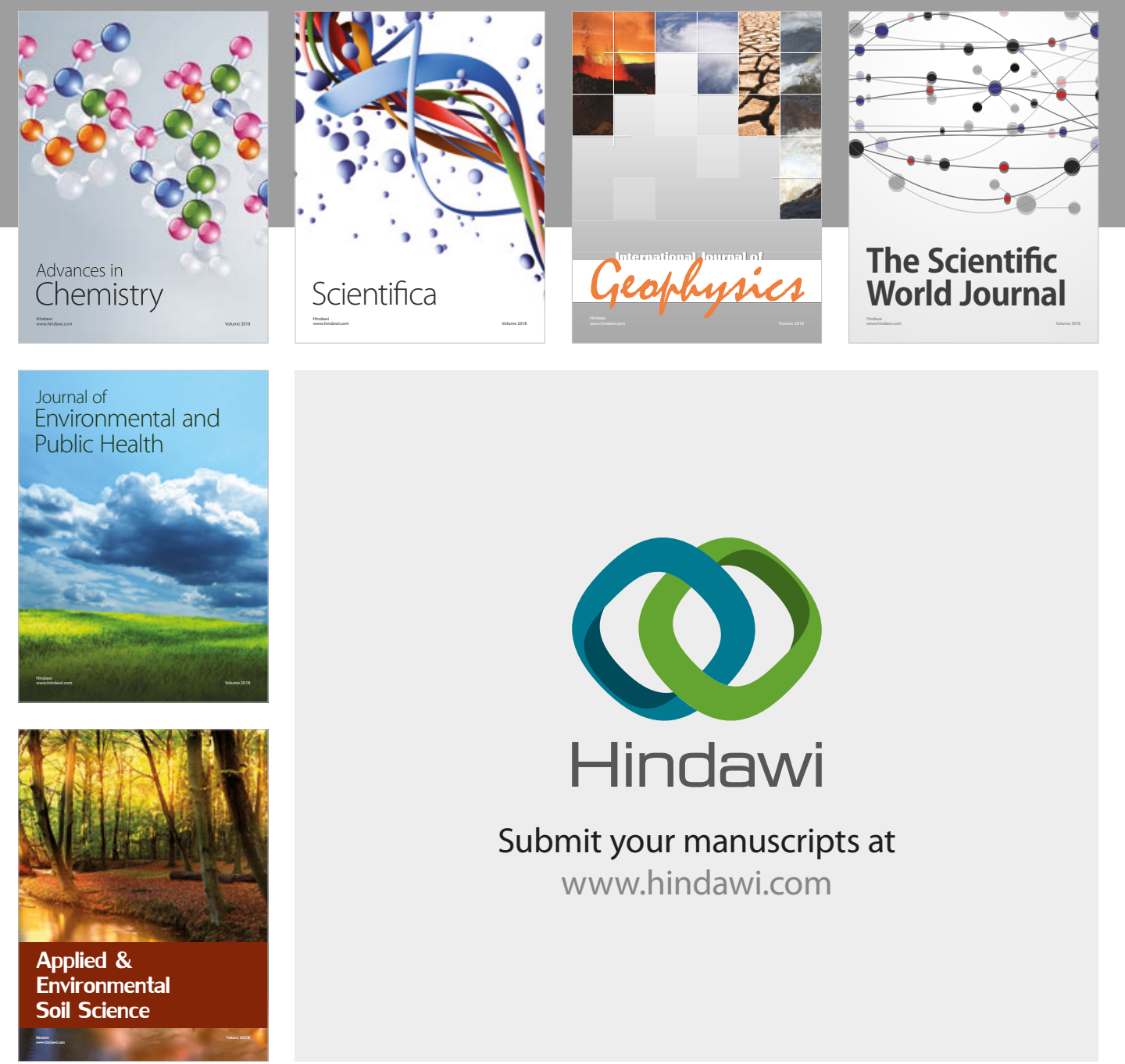

The Scientific

\section{World Journal}
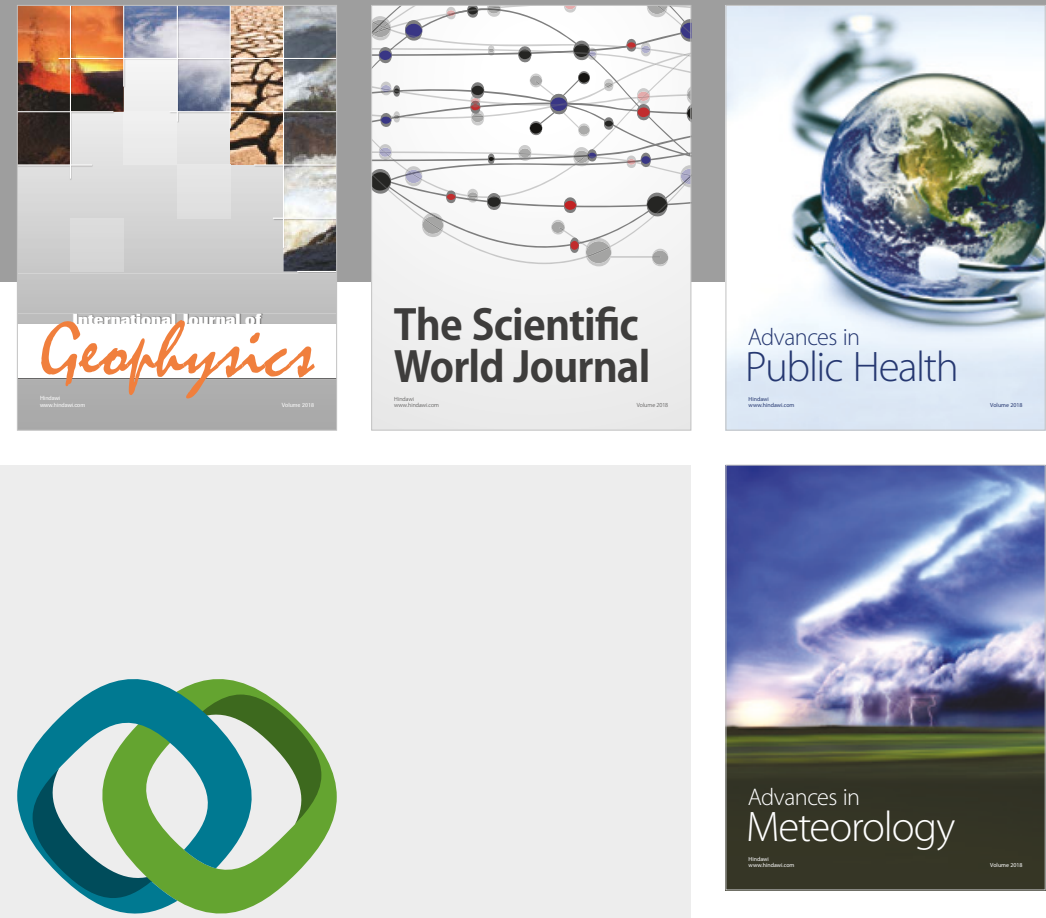

Advan

Public Health

\section{Hindawi}

Submit your manuscripts at

www.hindawi.com
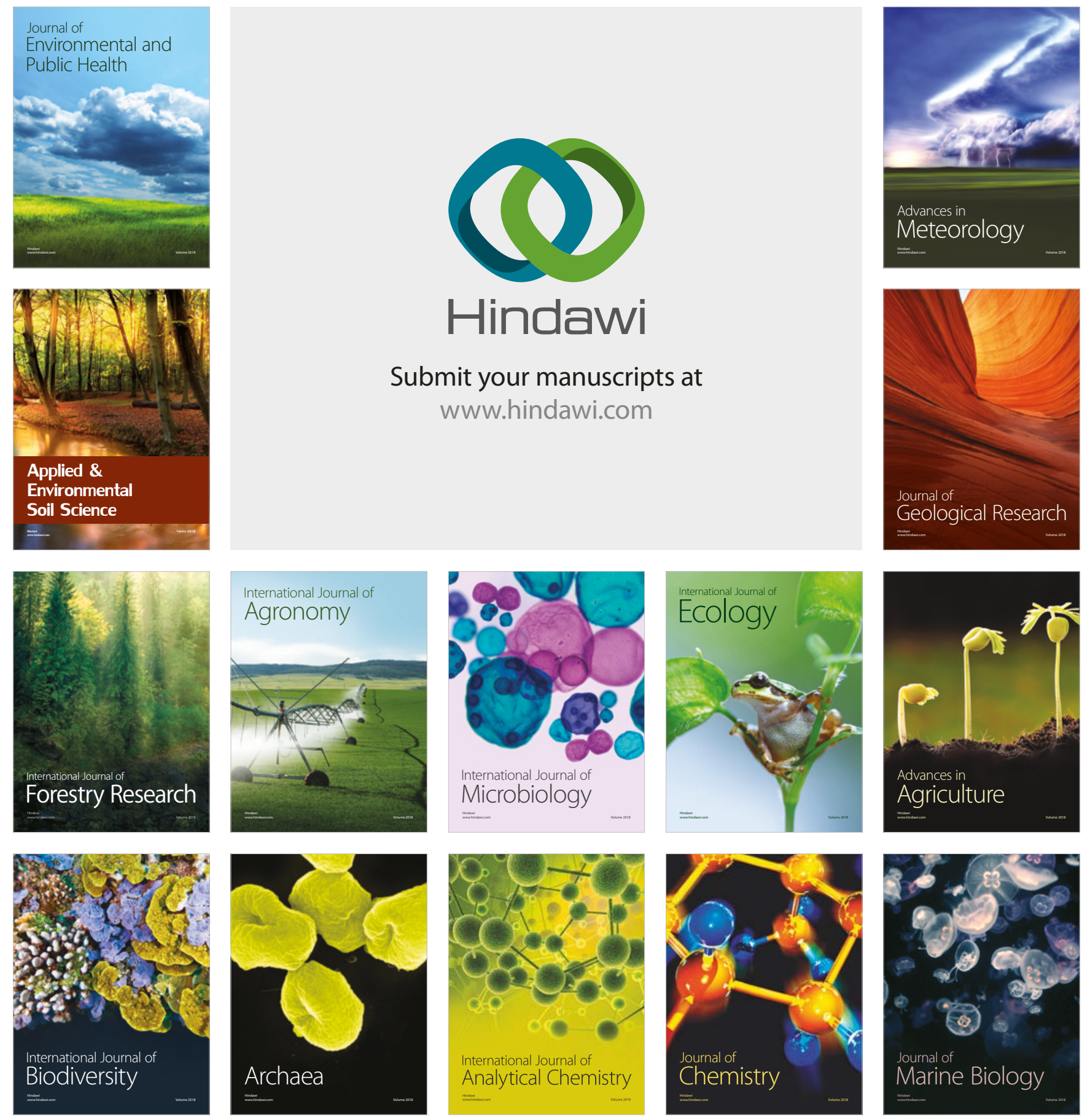